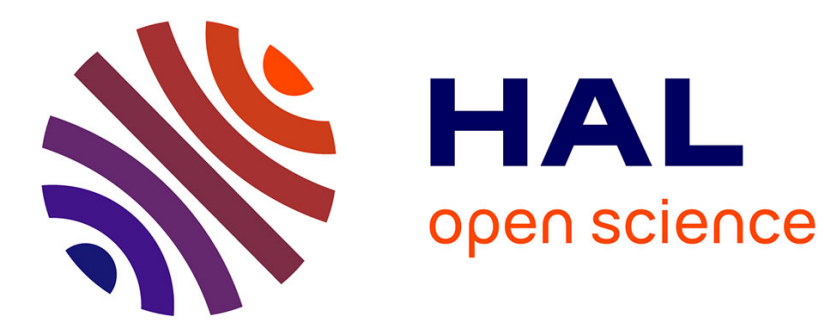

\title{
Robust circularly polarized few-optical-cycle solitons in Kerr media
}

Hervé Leblond, Houria Triki, François Sanchez, Dumitru Mihalache

\section{To cite this version:}

Hervé Leblond, Houria Triki, François Sanchez, Dumitru Mihalache. Robust circularly polarized fewoptical-cycle solitons in Kerr media. Physical Review A: Atomic, molecular, and optical physics [1990-2015], 2011, 83 (6), pp.063802. 10.1103/PhysRevA.83.063802 . hal-03187692

\section{HAL Id: hal-03187692 \\ https://univ-angers.hal.science/hal-03187692}

Submitted on 1 Apr 2021

HAL is a multi-disciplinary open access archive for the deposit and dissemination of scientific research documents, whether they are published or not. The documents may come from teaching and research institutions in France or abroad, or from public or private research centers.
L'archive ouverte pluridisciplinaire HAL, est destinée au dépôt et à la diffusion de documents scientifiques de niveau recherche, publiés ou non, émanant des établissements d'enseignement et de recherche français ou étrangers, des laboratoires publics ou privés. 


\title{
Robust circularly polarized few-optical-cycle solitons in Kerr media
}

\author{
Hervé Leblond, ${ }^{1}$ Houria Triki, ${ }^{2}$ François Sanchez, ${ }^{1}$ and Dumitru Mihalache ${ }^{1,3,4}$ \\ ${ }^{1}$ Laboratoire de Photonique d'Angers, EA 4464, Université d'Angers, 2 Bd. Lavoisier, \\ FR-49045 Angers Cedex 01, France \\ ${ }^{2}$ Radiation Physics Laboratory, Department of Physics, Faculty of Sciences, Badji Mokhtar University, \\ P.O. Box 12, DZ-23000 Annaba, Algeria \\ ${ }^{3}$ Horia Hulubei National Institute for Physics and Nuclear Engineering (IFIN-HH), 407 Atomistilor, \\ Magurele-Bucharest, RO-077125, Romania \\ ${ }^{4}$ Academy of Romanian Scientists, 54 Splaiul Independentei, Bucharest RO-050094, Romania \\ (Received 6 January 2011; revised manuscript received 8 April 2011; published 6 June 2011)
}

\begin{abstract}
We consider the propagation of circularly polarized few-cycle pulses (FCPs) in Kerr media beyond the slowly varying envelope approximation. Assuming that the frequency of the transition is far above the characteristic wave frequency (long-wave-approximation regime), we show that propagation of FCPs, taking into account the wave polarization, is described by the nonintegrable complex modified Korteweg-de Vries (cmKdV) equation. By direct numerical simulations, we get robust localized solutions to the cmKdV equation, which describe circularly polarized few-cycle-optical solitons and strongly differ from the breather soliton of the modified Korteweg-de Vries equation, which represents linearly polarized FCP solitons. The circularly polarized FCP soliton becomes unstable when the angular frequency is less than 1.5 times the inverse of the pulse length. The unstable subcycle pulses decay into linearly polarized half-cycle pulses, the polarization direction of which slowly rotates around the propagation axis.
\end{abstract}

DOI: 10.1103/PhysRevA.83.063802

PACS number(s): 42.65.Tg, 05.45.Yv, 42.65.Re

\section{INTRODUCTION}

Interest in intense ultrashort light pulses containing a few optical cycles has grown steadily in recent years since their first experimental realization in 1999. This mature research area has considerable potential for ultrafast optics applications in metrology of ultrafast phenomena and in systems performing laser ablation (micromachining, etching, microsurgery), among others. It still presents many exciting open problems from both a fundamental and an applied point of view. Notably, the ultrashort pulses possess extensive applications to the field of light-matter interactions, highorder harmonic generation, single-cycle nonlinear optics, and attosecond physics [1,2]; for a comprehensive review of earlier works in this area, see Ref. [3]. Recent progress in the study of the wave dynamics of few-cycle pulses (FCPs) in nonlinear optical media has paved the way for the development of new theoretical approaches to model their propagation in physical systems. Three classes of main dynamical models for FCPs have been put forward: (i) the quantum approach [4-6], (ii) the refinements within the framework of the slowly varying envelope approximation (SVEA) of the nonlinear Schrödinger-type envelope equations [7], and the non-SVEA models [8-12].

Other recent works on FCPs deal with few-cycle light bullets created by femtosecond filaments [13], the study of ultrashort spatiotemporal optical solitons in quadratic nonlinear media [14], the ultrashort spatiotemporal optical pulse propagation in cubic (Kerr-type) media without the use of the SVEA [15], and the possibility of generating few-cycle dissipative optical solitons [16]. The propagation of linearly polarized (LP) or scalar FCPs in Kerr media can be described beyond the SVEA by using the modified Kortewegde Vries (mKdV) [9], sine-Gordon (sG) [10], or mKdV-sG equations [11]. The $\mathrm{mKdV}$ and $\mathrm{sG}$ equations are completely integrable by means of the inverse scattering transform (IST) method [17], whereas the $\mathrm{mKdV}-\mathrm{sG}$ equation is completely integrable only if some condition between its coefficients is satisfied [18].

Previous studies of FCPs beyond the SVEA were restricted to linear polarization; therefore, the investigation of other kinds of wave polarization effects are of great importance from both fundamental and applied points of view. For instance, the circular polarization is important, from both a fundamental point of view, since it is related to the spin of the photon, and for applications, e.g., the circular dichroism of biological molecules such as DNA, circularly polarizing liquid crystals, etc.

We therefore focus on the vectorial nature of the electric field by applying the reductive perturbation method (multiscale analysis) to the Maxwell-Bloch equations. We mention that other vectorial non-SVEA models have been also proposed $[19,20]$, however, they were not carefully justified from a physical point of view, but only built from an analogy with common SVEA models.

The paper is organized as follows. In the following section, we introduce a governing model for an amorphous (glass) optical medium by considering a set of two-level atoms. In Sec. III, we derive the evolution equations in the long-wave approximation, describing the propagation of vector femtosecond optical solitons. In Sec. IV, we get robust circularly polarized (CP) few-cycle pulses and we analyze in detail their unique features. Section $\mathrm{V}$ describes the transition of unstable circularly polarized few-optical-cycle solitons to stable linearly polarized single-humped (half-cycle) pulses. Finally, in Sec. VI, we summarize the results of our analysis and we indicate some possible extensions of this work. 


\section{GOVERNING MODEL FOR AN AMORPHOUS MEDIUM}

As a simple model for a glass system, we consider a set of two-level atoms with Hamiltonian $H_{0}$,

$$
H_{0}=\hbar\left(\begin{array}{cc}
\omega_{a} & 0 \\
0 & \omega_{b}
\end{array}\right)
$$

where $\Omega=\omega_{b}-\omega_{a}>0$ is the frequency of the transition. The atoms may present some induced dipolar electric momentum $\vec{\mu}$, which is oriented randomly in space. By assuming a propagation along the $z$ axis, we can omit the component of $\vec{\mu}$ along the propagation direction $z$, and, thus, $\vec{\mu}=$ $\mu\left(\cos \theta \vec{e}_{x}+\sin \theta \vec{e}_{y}\right)$, with $\vec{e}_{x}$ and $\vec{e}_{y}$ being the unitary vectors along the $x$ and $y$ axis, respectively, and

$$
\mu=\left(\begin{array}{cc}
0 & \mu \\
\mu^{*} & 0
\end{array}\right) .
$$

The evolution of the electric field $\vec{E}$ is governed by the Maxwell equations, which, in the absence of magnetic effects and assuming a plane wave propagating along the $z$ axis, reduce to $\partial_{z}^{2} \vec{E}=c^{-2} \partial_{t}^{2}(\vec{E}+4 \pi \vec{P})$, where $\vec{P}$ is the polarization density. It is given by $\vec{P}=N\langle\operatorname{Tr}(\rho \vec{\mu})\rangle$, where $N$ is the number of atoms per unit volume, $\rho$ is the density matrix, and $\langle\cdot\rangle$ denotes the averaging over all directions in the $x-y$ plane.

The evolution of the density matrix is governed by the Heisenberg equation $i \hbar \partial_{t} \rho=[H, \rho]$, where $H=H_{0}-\vec{\mu} \cdot \vec{E}$ describes the coupling between the atoms and the electric field. The relaxation effects can be neglected here as in the scalar approximation (see [10]). The physical values of the relaxation times are indeed in the picosecond range, or even slower, thus, very large with regard to the pulse duration, which allows us to neglect them.

\section{EVOLUTION EQUATIONS FOR CP FCPS IN THE LONG-WAVE APPROXIMATION}

The typical frequency $\omega_{w}$ of the wave must be far away from the resonance frequency $\Omega$ because the transparency of the medium is required for soliton propagation. In this paper, we consider $\omega_{w} \ll \Omega$. The typical duration of the wave, say, $t_{w}=1 / \omega_{w}$, is very large with respect to the characteristic time $t_{r}=1 / \Omega$ associated to the transition. Thus, we are working in the long-wave approximation regime, as defined in the framework of the reductive perturbation method $[21,22]$. Next, we introduce a small parameter $\varepsilon=1 /\left(\Omega t_{w}\right)$, and the slow variables $\tau=\varepsilon(t-z / V), \zeta=\varepsilon^{3} z$. The retarded time variable $\tau$ describes the pulse shape, propagating at speed $V$ in a first approximation. Its order of magnitude $\varepsilon$ gives an account of the long-wave approximation $t_{w} \gg t_{r}$. The $\zeta$ variable of order $\varepsilon^{3}$ describes long-distance propagation according to the general theory of the derivation of KdV-type equations [22].

The electric field $\vec{E}$, the polarization density $\vec{P}$, and the density matrix $\rho$ are expanded in power series of $\varepsilon$ as $\vec{E}=$ $\sum_{n \geqslant 1} \varepsilon^{n}\left(u_{n}, v_{n}, 0\right), \vec{P}=\sum_{n \geqslant 1} \varepsilon^{n}\left(P_{n}, Q_{n}, 0\right), \rho=\sum_{n \geqslant 0} \varepsilon^{n} \rho_{n}$, in which the triplets of coordinates are given in the $(x, y, z)$ frame, and the profiles $u_{1}, v_{1}$, etc., are functions of the slow variables $\tau$ and $\zeta$. The components of $\rho$ are denoted by

$$
\rho=\left(\begin{array}{cc}
\rho_{a} & \rho_{t} \\
\rho_{t}^{*} & \rho_{b}
\end{array}\right) \text {. }
$$

We assume that, in the absence of wave, all atoms are in the fundamental state $(a)$ and, hence, all elements of $\rho_{0}$ are zero except $\rho_{0 a}=1$.

At lowest order $\varepsilon^{1}$, the Heisenberg equation yields $\rho_{1 t}=$ $\mu /(\hbar \Omega)\left(u_{1} \cos \theta+v_{1} \sin \theta\right)$. The polarization density is $\vec{P}=$ $(P, Q)$ with $P=N\left\langle\rho_{t} \mu^{*} \cos \theta+\right.$ c.c. $\rangle, Q=N\left\langle\rho_{t} \mu^{*} \sin \theta+\right.$ c.c. $\rangle$, where c.c. denotes the complex conjugate. As a result, we get $\left(P_{1}, Q_{1}\right)=N|\mu|^{2} /(\hbar \Omega)\left(u_{1}, v_{1}\right)$.

The Maxwell equation at leading order $\varepsilon^{3}$ gives the value of the velocity $V=c / n$, with the refractive index $n=\sqrt{1+4 \pi N|\mu|^{2} /(\hbar \Omega)}$. The expression of $n$ coincides with that obtained in the scalar model [10] if we take into account the fact that, for a linear polarization in the present framework, only one half of the dipoles would be active, being roughly aligned with the electric field. At the order $\varepsilon^{2}$, the Heisenberg equation yields $\rho_{1 a}=\rho_{1 b}=0$ and $\rho_{2 t}=\mu /(\hbar \Omega)\left(u_{2} \cos \theta+v_{2} \sin \theta\right)-i \mu /\left(\hbar \Omega^{2}\right) \partial_{\tau}\left(u_{1} \cos \theta+\right.$ $\left.v_{1} \sin \theta\right)$. Consequently, we get similar expressions for $P_{2}$ and $Q_{2}$ as those for $P_{1}$ and $Q_{1}$, with the only difference that $u_{1}$ and $v_{1}$ are replaced by $u_{2}$ and $u_{2}$. The Maxwell equation at order $\varepsilon^{4}$ is automatically satisfied.

At order $\varepsilon^{3}$, the Heisenberg equation gives rise to $\rho_{2 b}-$ $\rho_{2 a}=2|\mu|^{2} /\left(\hbar^{2} \Omega^{2}\right)\left(u_{1} \cos \theta+v_{1} \sin \theta\right)^{2}$, and a corresponding much longer expression for $\rho_{3 t}$. By using $\left\langle\cos ^{4} \theta\right\rangle=3 / 8$, $\left\langle\cos ^{2} \theta \sin ^{2} \theta\right\rangle=1 / 8$, etc., the expressions for the polarization density components $P_{3}$ are

$$
P_{3}=\frac{N|\mu|^{2}}{\hbar \Omega} u_{3}-\frac{N|\mu|^{2}}{\hbar \Omega^{3}} \partial_{\tau}^{2} u_{1}-\frac{3 N|\mu|^{4}}{2 \hbar^{3} \Omega^{3}}\left(u_{1}^{2}+v_{1}^{2}\right) u_{1},
$$

and analogously for $Q_{3}$. Next, the Maxwell equation at order $\varepsilon^{5}$ yields the following pair of coupled equations:

$$
\begin{gathered}
\partial_{\zeta} u_{1}=A \partial_{\tau}^{3} u_{1}+B \partial_{\tau}\left[\left(u_{1}^{2}+v_{1}^{2}\right) u_{1}\right], \\
\partial_{\zeta} v_{1}=A \partial_{\tau}^{3} v_{1}+B \partial_{\tau}\left[\left(u_{1}^{2}+v_{1}^{2}\right) v_{1}\right],
\end{gathered}
$$

in which we have set $A=2 \pi N|\mu|^{2} /\left(n c \hbar \Omega^{3}\right), \quad B=$ $3 \pi N|\mu|^{4} /\left(n c \hbar^{3} \Omega^{3}\right)$. As in the scalar model [10], the dispersion and nonlinear coefficients $A$ and $B$ express as $A=$ $n^{\prime \prime} / 2 c$, where $n^{\prime \prime}$ is the second derivative of the refractive index with respect to $\omega$, and $B=(-6 \pi / n c) \chi^{(3)}$, in which $\chi^{(3)}=\chi_{x x x x}^{(3)}(\omega ; \omega, \omega,-\omega)$ is the relevant nonlinear susceptibility. $n^{\prime \prime}$ and $\chi^{(3)}$ should be evaluated for frequencies far below the resonance line. The fact that the nonlinear term involves the intensity $\left(u_{1}^{2}+v_{1}^{2}\right)$ directly reflects the isotropy of the model. Recall that, in an isotropic medium as a glass and, as in this paper, the other component of $\chi^{(3)}$ satisfies $\chi_{x x y y}^{(3)}(\omega ; \omega, \omega,-\omega)=\chi_{x y x y}^{(3)}(\omega, \omega, \omega,-\omega)=$ $\chi_{x y y x}^{(3)}(\omega, \omega, \omega,-\omega)=(1 / 3) \chi_{x x x x}^{(3)}(\omega ; \omega, \omega,-\omega)$.

Equations (5) and (6) can be written in the normalized form as

$$
\begin{aligned}
& U_{Z}=U_{T T T}+\left[\left(U^{2}+V^{2}\right) U\right]_{T}, \\
& V_{Z}=V_{T T T}+\left[\left(U^{2}+V^{2}\right) V\right]_{T},
\end{aligned}
$$

where the subscripts $Z$ and $T$ denote the derivatives, and the functions and variables are defined as $U=u_{1} / \mathcal{E}, V=v_{1} / \mathcal{E}$, $Z=z / \mathcal{L}, T=(t-z / V) / t_{w}$, with $\mathcal{L}=2 c t_{w}^{3} / n^{\prime \prime}$ and $\mathcal{E}=$ $\left(1 / 2 t_{w}\right) \sqrt{n n^{\prime \prime} /\left(-3 \pi \chi^{(3)}\right)}$. Equations (7) and (8) are a set of coupled $\mathrm{mKdV}$ equations describing the propagation of optical FCPs in an amorphous medium presenting cubic nonlinearity 
and dispersion. They can be also seen as describing the interaction of two linearly polarized (LP) FCPs, $U$ and $V$.

The above approach can be easily extended to the case of two-level medium, in which the excited level is degenerated twice, with the induced dipole oriented either in the $x$ or in the $y$ direction. As a final result, we get the same set of equations (5) and (6) as above, with very slightly modified coefficients. In fact, in this case, the coefficients $A$ and $B$ have exactly the same expressions as in the scalar model [10].

Setting $f=U+i V$, Eqs. (7) and (8) reduce to $f_{Z}=$ $f_{T T T}+\left(|f|^{2} f\right)_{T}$, which is known as the complex modified Korteweg-de Vries (cmKdV) equation. Confusion must be avoided between this equation and the other $\mathrm{cmKdV}$ equation $f_{Z}=f_{T T T}+\left(|f|^{2}\right) f_{T}$. Indeed, the latter is completely integrable [23], while the former is not. These two equations are sometimes referred to as cmKdV I and cmKdV II equations, respectively. The integrable equation has been extensively studied (see, e.g., [23,24]), while fewer studies have been devoted to the nonintegrable one [25]. In the frame of the optics of FCPs, the field $f$ must vanish at infinity. With this condition, the cmKdV I equation does not admit any exact analytical solution apart from those which exactly coincide with the solutions of the real $\mathrm{mKdV}$ equation. Indeed, setting $f=u e^{i \varphi}$, with $u=u(Z, T)$ and $\varphi$ a constant, reduces the cmKdV I equation to the real one. All LP FCP solitons are retrieved in this way. Their stability to a random perturbation of the polarization can be tested numerically. If we add to the constant $\varphi$ a random noise (we used an amplitude of $0.1 \times 2 \pi)$, it is obtained that the pulse is not destroyed and its polarization remains linear. However, the direction of the linear polarization slowly rotates around the propagation direction. A more interesting situation would be a CP soliton of the form $f=u(T-w Z) e^{i(\omega T-k Z)}$. A simple analysis shows that no exact, even numerical, steady-state solution of this type exists. However, solutions having approximately the above waveform exist and are very robust.

\section{ROBUST CP FEW-OPTICAL-CYCLE SOLITONS}

An approximate solution to the cmKdV I equation is

$$
f=b \sqrt{6} \operatorname{sech}\left[b\left(T-3 \omega^{2} Z\right)\right] e^{i \omega\left[T-\left(\omega^{2}-3 b^{2}\right) Z\right]},
$$

which is valid for long pulses $(b \ll \omega)$. The numerical resolution of the cmKdV equation is performed using the exponential time differencing second-order Runge-Kutta method [26]. Due
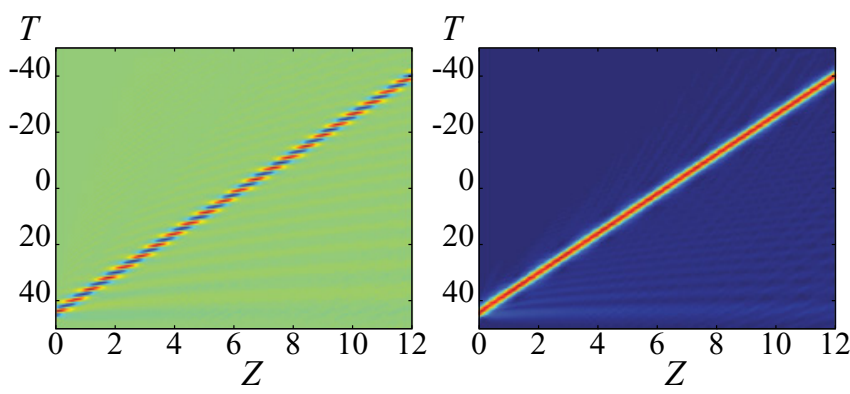

FIG. 1. (Color online) Propagation of a CP FCP. Left panel: $x$-polarized component $U$. Right panel: Norm of the electric field $|f|$. Initial data are given by Eq. (9) with $b=1$ and $\omega=2$.

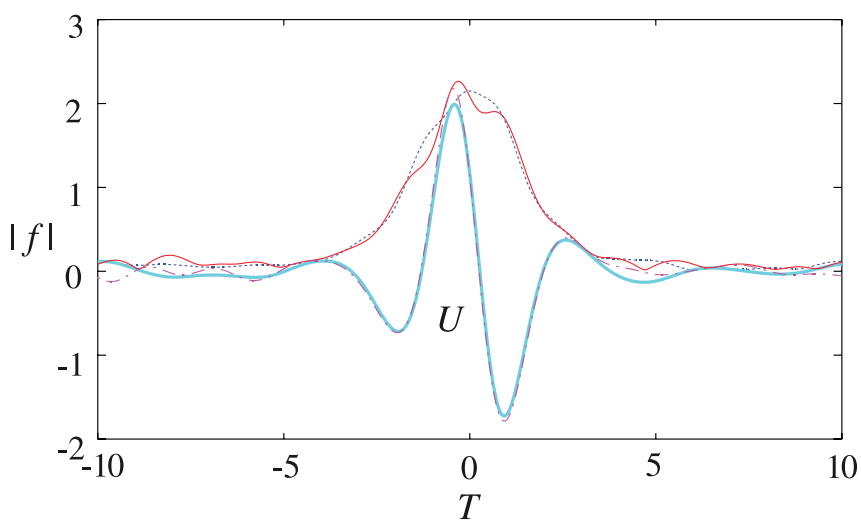

FIG. 2. (Color online) Initial $(Z=100)$ and final $(Z=10000)$ profiles of the FCP plotted on Fig. 1 for the input given by Eq. (9). Blue (dotted line): initial $|f|$; light blue (thick gray line): initial $U$; red (thin solid line): final $|f|$; pink (dashed-dotted line): final $U$.

to the scale invariance of the cmKdV equation, only the ratio $b / \omega$ may modify the stability properties of the solution. Practically, we fix $b=1$ and decrease the frequency $\omega$.

Figures 1 and 2 show the evolution of a FCP of this form, with $b=1$ and $\omega=2$. The locations and phase have been artificially reset to the initial value so that comparison can be made easily. The robustness of the FCP is obvious, and its width and maximum amplitude are conserved. The effect of dispersion is investigated by numerical computation: the pulse duration (full width at half maximum, FWHM) increases linearly with propagation distance. The dispersion length $z_{D}$, defined as the distance at which the FWHM has been mulitiplied by $\sqrt{2}$, is very small, less than 0.08 for the data of Figs. 1 and 2. The propagation distance $z=10000$ thus corresponds to about $1.3 \times 10^{5}$ dispersion lengths.

However, its shape is somehow distorted after propagation. The propagation speed is also quite different from the result

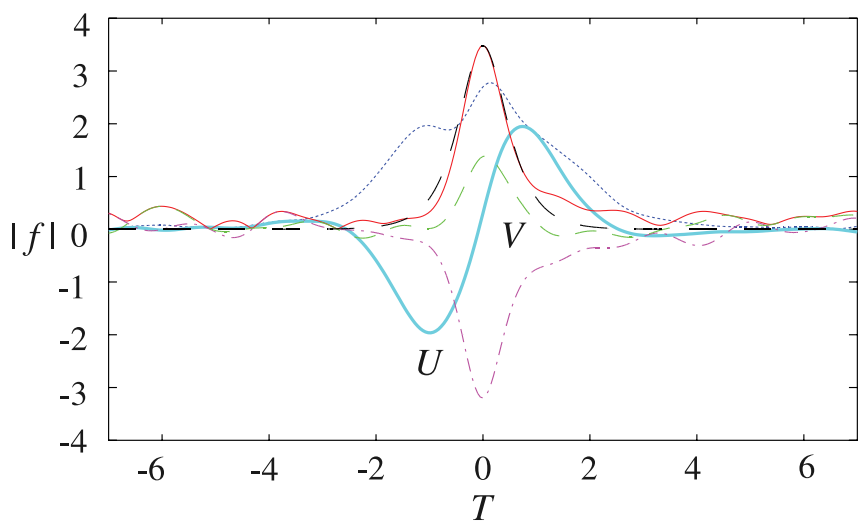

FIG. 3. (Color online) Initial $(Z=100)$ and final $(Z=10000)$ profiles for an unstable CP FCP. Initial data are defined by the breather of the real $\mathrm{mKdV}$ equation with both pulse width and angular frequency equal to 1 , for the component $U$, and the same with a $\pi / 2$ dephasing for $V$. Blue (dotted line): initial $|f|$; light blue (thick gray line): initial $U=\operatorname{Re}(f)$; red (thin solid line): final $|f|$; pink (dashed-dotted line): final $U$; green (short dashed line): final $V=\operatorname{Im}(f)$; black (long dashed line): fit of the final $|f|$ by a fundamental soliton of the real $\mathrm{mKdV}$ equation. 

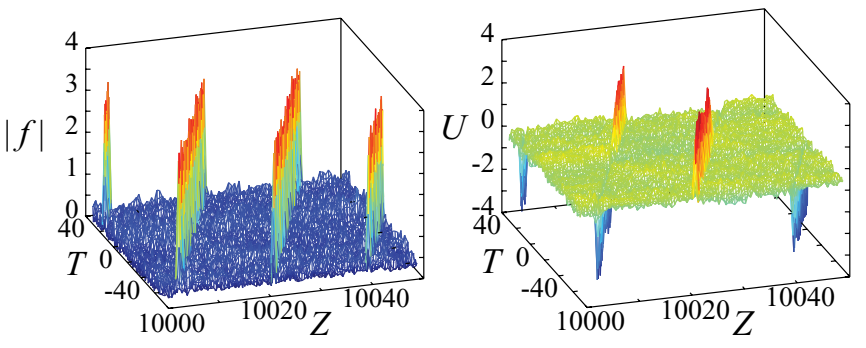

FIG. 4. (Color online) Propagation of the slowly rotating LP half-cycle pulse resulting from the instability of the CP FCP with both angular frequency and pulse length 1. Left panel: Norm of the electric field $|f|$. Right panel: $x$-polarized component $U$. The pulse crosses the computation box several times due to periodic boundary conditions.

of the above analytical approximate solution. In fact, since no steady state with linear phase exists, the pulse is not a true steady state and, consequently, its velocity is not constant during the propagation. It seems to oscillate around the linear group velocity during propagation, however, it can not be evaluated from numerical data due to eventual stroboscopic effect with respect to the discretization frequency. Nevertheless, the pulse is very robust. Notice that the approximate solution (9) does not have a zero mean value, except at the SVEA limit $b \ll \omega$. However, the mean value of the field is conserved. It is likely that the CP FCP soliton would have a zero mean value and, hence, this would explain the discrepancy between the approximate analytical solution (9) and the direct numerical computation shown in Fig. 1. We actually checked this interpretation by considering an input having zero mean value. Such an alternative input can be built using the breather (or two-soliton) solution of the real $\mathrm{mKdV}$ equation [27].

\section{TRANSITION TO A HALF-CYCLE SOLITON}

We have found that the value $\omega / b \simeq 1.5$ is the lower limit for the stability of the CP FCP soliton. For smaller values of the ratio $\omega / b$, the FCP becomes unstable and decays into a LP single-humped (half-cycle) pulse in the form of a fundamental soliton of the real $\mathrm{mKdV}$. The transition occurs at about $Z \simeq 19100,9300$, or 400 , for for $\omega / b=1.4$, 1.3 , and 1 , respectively. Further, it occurs very abruptly and involves a strong modification of the optical spectrum of the unstable CP FCP. The result of the transition is a single pulse, the profile of which accurately coincides with that of the fundamental soliton solution to the real $\mathrm{mKdV}$ equation $U=\sqrt{2} b \operatorname{sech}\left(b T-b^{3} Z\right)$, as shown in Fig. 3, but which slowly rotates around the propagation axis. Figure 4 shows the $z$ dependence of $|f|$ and the oscillations of the polarization component $U$. The period of oscillation is close to the length of the propagation distance interval presented in the figure, about 50 , which yields an angular frequency $\omega \simeq 2 \pi / 50 \simeq 0.13$. In some sense, $\omega$ has fallen about one order of magnitude. However, this should not be considered as a frequency change since $\omega$ no longer represents the central frequency of the pulse. This rotation speed is not modified during the propagation from $Z=2000$ to 10000 , at least. However, it strongly depends on the continuous pedestal. Indeed, if we removed this pedestal by setting the field $f$ to zero out of the main pulse and then computed the evolution, the rotation does not appear any more. As a final remark, we note that half-cycle optical solitons were also put forward in quadratic nonlinear media [28].

\section{CONCLUSIONS}

In conclusion, by using the multiscale perturbation analysis in the long-wave-approximation regime, we derived approximate evolution equations governing the propagation of femtosecond optical solitons in cubic nonlinear media beyond the SVEA, taking into account the vectorial character of the electric field. We found that the interacting waveforms are adequately described by a coupled pair of $\mathrm{mKdV}$ equations or, equivalently, by the nonintegrable complex mKdV equation. Numerical and approximate analytical approaches show the existence of soliton solutions, which are very different from the breather of the real $\mathrm{mKdV}$ equation that accounts for the linearly polarized FCP solitons, in the sense that their amplitudes yield a single hump, even for a pulse containing several optical cycles. Thus, found solitons describe circularly polarized FCPs, which are shown to be very robust. However, the circularly polarized FCP solitons become unstable for subcycle pulses. We show that the instability threshold corresponds to a ratio $\omega / b \simeq 1.5$ of the angular frequency divided by the inverse of the pulse length. Below this threshold, the unstable subcycle pulses decay into linearly polarized half-cycle pulses, the polarization direction of which slowly rotates around the propagation axis. Challenging extensions suggested by this paper are to consider (i) the so-called short-wave approximation in which the resonance frequency of the atoms is below the optical frequencies, and (ii) the case of two transitions, one below and one above the range of propagated wavelengths. We finally mention that another interesting issue is the generalization of this paper to one or even to two spatial transverse dimensions, in addition to time and spatial longitudinal coordinates.

\section{ACKNOWLEDGMENT}

The work of DM in Angers was supported by a grant from the Région Pays de Loire, France.
[1] E. Goulielmakis et al., Science 320, 1614 (2008).

[2] A. Scrinzi, M. Yu. Ivanov, R. Kienberger, and D. M. Villeneuve, J. Phys. B: At. Mol. Opt. Phys. 39, R1 (2006).

[3] T. Brabek and F. Krausz, Rev. Mod. Phys. 72, 545 (2000).

[4] N. N. Rosanov, V. E. Semenov, and N. V. Vyssotina, Laser Phys. 17, 1311 (2007).
[5] A. Nazarkin, Phys. Rev. Lett. 97, 163904 (2006).

[6] A. I. Maimistov, Quantum Electron. 40, 756 (2010).

[7] T. Brabec and F. Krausz, Phys. Rev. Lett. 78, 3282 (1997).

[8] E. M. Belenov and A. V. Nazarkin, Pis'ma Zh. Eksp. Teor. Fiz. 51, 252 (1990) [JETP Lett. 51, 288 (1990)]; A. I. Maimistov and S. O. Elytin, J. Mod. Opt. 39, 2201 (1992); A. E. Kaplan 
and P. L. Shkolnikov, Phys. Rev. Lett. 75, 2316 (1995); S. V. Sazonov, Zh. Eksp. Teor. Fiz. 119, 419 (2001) [JETP 92, 361 (2001)].

[9] I. V. Mel'nikov, D. Mihalache, F. Moldoveanu, and N.-C. Panoiu, Phys. Rev. A 56, 1569 (1997); Pis'ma Zh. Eksp. Teor. Fiz. 65, 380 (1997) [JETP Lett. 65, 393 (1997)]; I. V. Mel'nikov, D. Mihalache, and N.-C. Panoiu, Opt. Commun. 181, 345 (2000); I. V. Mel'nikov, H. Leblond, F. Sanchez, and D. Mihalache, IEEE J. Sel. Top. Quantum Electron. 10, 870 (2004).

[10] H. Leblond and F. Sanchez, Phys. Rev. A 67, 013804 (2003).

[11] H. Leblond, S. V. Sazonov, I. V. Mel'nikov, D. Mihalache, and F. Sanchez, Phys. Rev. A 74, 063815 (2006); H. Leblond, I. V. Mel'nikov, and D. Mihalache, ibid. 78, 043802 (2008); H. Leblond and D. Mihalache, ibid. 79, 063835 (2009).

[12] Sh. Amiranashvili, A. G. Vladimirov, and U. Bandelow, Phys. Rev. A 77, 063821 (2008).

[13] L. Bergé and S. Skupin, Phys. Rev. Lett. 100, 113902 (2008).

[14] H. Leblond, D. Kremer, and D. Mihalache, Phys. Rev. A 80, 053812 (2009).

[15] H. Leblond, D. Kremer, and D. Mihalache, Phys. Rev. A 81, 033824 (2010); H. Leblond and D. Mihalache, ibid. 81, 063815 (2010).
[16] N. N. Rosanov, V. V. Kozlov, and S. Wabnitz, Phys. Rev. A 81, 043815 (2010); H. Leblond and D. Mihalache, J. Phys. A: Math. Theor. 43, 375205 (2010).

[17] R. K. Dodd, J. C. Eilbeck, J. D. Gibbon, and H. C. Morris, Solitons and Nonlinear Wave Equations (Academic, London, 1982).

[18] K. Konno, W. Kameyama, and H. Sanuki, J. Phys. Soc. Jpn. 37, 171 (1974).

[19] M. Pietrzyk, I. Kanattšikov, and U. Bandelow, J. Nonlin. Math. Phys. 15, 162 (2008).

[20] V. G. Bespalov, S. A. Kozlov, Yu. A. Shpolyansky, and I. A. Walmsley, Phys. Rev. A 66, 013811 (2002).

[21] T. Taniuti and C.-C. Wei, J. Phys. Soc. Jpn. 24, 941 (1968).

[22] H. Leblond, J. Phys. B: At. Mol. Opt. Phys. 41, 043001 (2008).

[23] M. V. Foursov, J. Math. Phys. 41, 6173 (2000).

[24] B. A. Malomed, J. Fujioka, A. Espinosa-Ceron, R. F. Rodriguez, and S. González, Chaos 16, 013112 (2006).

[25] A. A. Mohammad and M. Can, J. Phys. A: Math. Gen. 28, 3223 (1995).

[26] S. M. Cox and P. C. Matthews, J. Comput. Phys. 176, 430 (2002).

[27] R. Hirota, Lect. Notes Math. 515, 40 (1976).

[28] H. Leblond, Phys. Rev. A 78, 013807 (2008). 